\title{
Lithium therapy and its interactions
}

\section{SUMMARY}

Lithium is one of the most effective mood stabilisers for people with a mood disorder. However, many of these patients are also taking other medicines that could potentially interact with lithium.

To minimise the risk of relapse, it is usually necessary to maintain the lithium serum concentration between $0.6 \mathrm{mmol} / \mathrm{L}$ and $0.8 \mathrm{mmol} / \mathrm{L}$.

Lithium clearance is easily influenced by drugs that alter renal function such as ACE inhibitors, angiotensin receptor antagonists, diuretics, and non-steroidal anti-inflammatory drugs.

It is therefore prudent for prescribers to monitor and adjust the lithium dose to avoid adverse effects or loss of efficacy.

\section{Introduction}

Driven by new research reinforcing the unique benefits of lithium, there has been a worldwide resurgence in the prescription of lithium. In clinical practice it is used predominantly to stabilise mood. It remains one of the most effective options for bipolar disorder, ${ }^{2}$ along with the newer atypical antipsychotics. ${ }^{3}$ Lithium also serves as an effective adjunctive option for recurrent or resistant major depressive disorder and has anti-suicidal properties which are invaluable in the management of mood disorders.

Lithium is simple to administer and is usually well tolerated. Routine management of patients receiving lithium monotherapy is relatively straightforward. ${ }^{4}$ However, complications can arise when other drugs are added that could potentially interact with lithium. Regular monitoring of lithium plasma concentrations and other safety parameters is essential. Results should be communicated to the patient and everyone involved in their care. Aids are available to assist prescribers with lithium management, including an Australian tool called the 'Lithiumeter'. ${ }^{4}$

\section{Indications for lithium}

Patients with classic, episodic and remitting bipolar disorder with a family history and no psychiatric comorbidity are most likely to respond to lithium. Typically, lithium is effective in about a third of patients - with response rates up to two-thirds in those whose relatives have achieved good responses. ${ }^{5}$ It is likely that people who commence lithium early in the course of their illness may have greater likelihood of response. In major depressive disorder, lithium is used to augment antidepressant drugs.

\section{Lithium monitoring}

Lithium has a very narrow therapeutic window for maintenance therapy. Too little lithium risks undertreatment of the mood disorder and increases the risk of relapse. Too much lithium increases the risk of both acute and chronic toxicity. Lithium concentrations should always be measured 12 hours after the last dose.

For the maintenance phase of treatment, recent guidelines recommend that patients maintain a serum concentration of $0.6-0.8 \mathrm{mmol} / \mathrm{L}$ to maximise therapeutic benefit. ${ }^{6}$ For acute treatment in mania, serum concentrations should be increased to $0.6-1.0 \mathrm{mmol} / \mathrm{L}$ as tolerated. In depression, concentrations can be in the range of $0.4-0.8 \mathrm{mmol} / \mathrm{L}$. In practice, target concentrations and monitoring practices are often inconsistent. Not all pathology laboratories use the same reference ranges, therefore noting whether the lithium concentration is consistent with the patient's presentation and the guidelines is essential.

As a part of optimising lithium dosing, clinicians may notice that a specific concentration achieves the most therapeutic benefit during euthymic periods and during manic and depressive episodes. Taking note of this is essential and helps to ensure stability of these patient-specific concentrations over time, particularly during each illness phase.

Maintenance of the therapeutic concentration (and adherence) is the strongest predictor of long-term stability. However, in some patients, stabilising their mood is not always possible with lithium alone. A trial with other mood stabilisers, such as adjunctive sodium valproate or an atypical antipsychotic, is often necessary.

\section{Gin S Malhi'1,2,3 \\ Erica Bell',2,3 \\ Tim Outhred ${ }^{1,2,3}$ \\ Michael Berk ${ }^{4,5,6,7}$}

1 Discipline of Psychiatry, Northern Clinical School, University of Sydney

2 Department of Academic Psychiatry, Northern Sydney Local Health District

${ }^{3}$ CADE Clinic, Royal North Shore Hospital, Northern Sydney Local Health District

${ }^{4}$ IMPACT - the Institute for Mental and Physical Health and Clinical Translation, School of Medicine, Barwon Health, Deakin University, Geelong, Vic

${ }^{5}$ Department of Psychiatry, University of Melbourne, Royal Melbourne Hospital

${ }^{6}$ Florey Institute for Neuroscience and Mental Health, University of Melbourne

${ }^{7}$ Orygen, The National Centre of Excellence in Youth Mental Health, Melbourne

\section{Keywords}

bipolar disorder, lithium, mood disorders

Aust Prescr 2020;43:91-3 https://doi.org/10.18773/ austprescr.2020.024

Corrected 12 June 2020

This is the corrected version of the article.

Correction notice available at: https://doi.org/10.18773/ austprescr.2020.041 


\section{Box Monitoring schedule for lithium therapy}

Baseline assessments and follow-up of patients should be performed:

- during the early maintenance phase (e.g. baseline, 7 days, 14 days and 28 days) then at 3,6 and 12 months, then annually

and

- when there are any changes in presentation

- following abnormal findings

- when altering the treatment regimen.

Regularly check the following:

- serum lithium concentrations and mood and stability over time

- renal function

- electrolytes, urea, creatinine

- estimated glomerular filtration rate

- thyroid and parathyroid function

- thyroid stimulating hormone

- calcium

- blood and cardiometabolic tests

- full blood count, glucose, lipids, liver function tests

- ECG

- weight, BMI, umbilical girth

- diet and eating behaviour

- exercise and hydration

- other comorbidities

- adverse effects

- cognition

- global functioning

- treatment adherence

Based on reference 4

\section{Table Major adverse effects of lithium therapy}

\begin{tabular}{|c|c|c|}
\hline Toxicity & Adverse effect & Action \\
\hline \multirow[t]{6}{*}{ Acute } & Any acute adverse effect or presentation & Measure lithium concentration \\
\hline & Headache, fatigue & Consider stopping lithium \\
\hline & Thirst, taste & Review medication \\
\hline & Arrythmias & Hospitalisation \\
\hline & Nausea, vomiting, diarrhoea, polyuria & $\begin{array}{l}\text { Review hydration and consider } \\
\text { haemodialysis }\end{array}$ \\
\hline & Tremor & Monitoring and review medication \\
\hline \multirow[t]{3}{*}{ Chronic } & $\begin{array}{l}\text { Cognitive effects, ataxia, agitation, } \\
\text { confusion, sluggishness }\end{array}$ & $\begin{array}{l}\text { Monitor changes, optimise lithium } \\
\text { concentrations, neurological referral }\end{array}$ \\
\hline & Thyroid or parathyroid dysfunction & $\begin{array}{l}\text { Monitor changes, optimise lithium } \\
\text { concentrations, endocrinology } \\
\text { referral }\end{array}$ \\
\hline & Renal dysfunction & $\begin{array}{l}\text { Monitor changes, optimise lithium } \\
\text { concentrations, nephrology referral }\end{array}$ \\
\hline
\end{tabular}

A recommended monitoring schedule for lithium in a patient not taking other drugs is outlined in the Box. ${ }^{4}$ Drug interactions are more likely to affect patients as they get older because of declining renal function and the accumulation of medical comorbidities. Close monitoring and dose adjustments are therefore often needed as patients get older.

In patients taking concomitant drugs, extra care should be taken because of the risk of drug interactions. Lithium concentrations should be closely monitored around the time of medication changes - at least just before and when the drugs have reached steady states. Lithium's half-life is about 24 hours, so a steady state is usually achieved after 5-7 days. A complete list of lithium drug interactions can be found at MIMS Online or Drugs.com.

Regular monitoring is required until a therapeutic concentration of lithium is reached and maintained, and any time that the patient presents with symptoms of lithium toxicity. ${ }^{7}$ Conditions leading to haemodynamic and volume changes such as dehydration, febrile illness, gastrointestinal loss, drug interactions, perioperative management and surgery can affect lithium serum concentrations and levels should be rechecked in these circumstances.

Prescribers should contact the treating psychiatrist or consult a medicines information pharmacist if they are unsure how to manage a patient. Having up-to-date serum lithium concentrations at hand will assist.

\section{Adverse effects of lithium}

Regular long-term monitoring of lithium concentrations is essential to avoid both acute and chronic toxicity. Physical examinations and laboratory investigations should be performed at baseline and regular intervals after that (see Box).

Common acute adverse effects include tremor, polydipsia, polyuria, dysgeusia, nausea and diarrhoea (see Table). Prescribers can reassure patients that these adverse effects are usually transient after starting treatment. They are often dependent on the serum concentration of lithium and frequently subside within days or weeks. Nephrogenic diabetes insipidus (polyuria and polydipsia) is a common adverse effect of lithium. Chronic adverse effects include subjective cognitive effects, thyroid and parathyroid dysfunction, and renal dysfunction (see Table). Some patients may report more mild neurocognitive effects such as 'brain fog', 'emotional greying', 'slowing', 'shakiness', anomia, and 'reduced creativity'. The higher the lithium concentration, the greater the risk of toxic presentations. In the long term, or with higher blood concentrations or repeated acute fluctuations, lithium leads to end-stage renal failure in $1 \%$ of patients (over 15 years treatment). ${ }^{8}$ However, it should be noted that most patients do not experience renal adverse effects. 


\section{Common drug-drug interactions with lithium}

The most common and noteworthy drug-drug interactions with lithium are pharmacokinetic in nature. The lithium ion is extensively absorbed in the gastrointestinal tract. The main determinant of serum concentrations is renal excretion, therefore the main drug interactions occur when co-administered drugs alter renal function, specifically modifying glomerular filtration and tubular reabsorption.

The most commonly prescribed drugs that have the potential to interact with lithium are ACE inhibitors, angiotensin II receptor antagonists (sartans), diuretics, and non-steroidal anti-inflammatory drugs (NSAIDs). Combinations of these are frequently used, so prescribers should be aware of their additive effects for a patient taking lithium.

\section{ACE inhibitors and angiotensin II receptor antagonists}

Several case reports and hospital admission studies have shown that ACE inhibitors and angiotensin II receptor antagonists can increase lithium serum concentrations and increase the chance of toxicity. Closer monitoring of lithium concentrations is needed when people start either of these drugs and the lithium dose will probably need to be reduced until a stable therapeutic concentration has been achieved. Closer monitoring is also required when these drugs are stopped.

\section{Diuretics}

When any diuretic is used, lithium concentrations must be carefully monitored. Thiazide and thiazidelike diuretics increase sodium reabsorption which decreases the clearance of lithium and significantly elevates lithium concentrations in serum. This is enough to fall out of the therapeutic range in many cases. As a rule of thumb, many prescribers halve the lithium dose then up- or down-titrate the dose with monitoring. Other prescribers avoid thiazide diuretics altogether. Amiloride is recommended as a diuretic because it blocks entry of lithium through the epithelial sodium channel in the collecting duct. This reduces lithium accumulation and may improve kidney function in patients on long-term treatment. ${ }^{9}$
Other diuretics such as the osmotic methylxanthine (e.g. theophylline) and loop (e.g. furosemide (frusemide)) and potassium-sparing (e.g. spironolactone) diuretics may also alter lithium concentrations.

\section{Non-steroidal anti-inflammatory drugs}

Patients on lithium therapy should be advised to avoid NSAIDs. Regular use is more problematic than episodic use. NSAIDs differentially alter lithium concentrations by multiple mechanisms, and one of these is to reduce prostaglandin E2 by inhibiting cyclo-oxygenase. This reduces vasodilation of the afferent arteriole which decreases blood flow to the glomerulus. This decreases glomerular filtration and consequently lithium excretion. If NSAIDs are indicated, they should be used under medical guidance with closer monitoring of lithium concentrations. Lower lithium doses may be required.

\section{Other drugs}

Acetazolamide for intraocular pressure, glaucoma and epilepsy has been shown to significantly increase lithium clearance.

\section{Conclusion}

Lithium has an important role in the treatment of mood disorders. Prescribers need to be mindful of its potential drug interactions and the impact they can have on patients. Improved knowledge of and confidence with monitoring will contribute to better patient outcomes. $<$

Gin Malhi has received grant or research support from the National Health and Medical Research Council, Australian Rotary Health, NSW Health, Ramsay Health, American Foundation for Suicide Prevention, Ramsay Research and Teaching Fund, Elsevier, AstraZeneca and Servier; has been a speaker for AstraZeneca, Janssen-Cilag, Lundbeck, Otsuka and Servier; and has been a consultant for AstraZeneca, Janssen Cilag, Lundbeck, Otsuka and Servier. Michael Berk is supported by a National Health and Medical Research Council Senior Principal Research Fellowship (1059660 and APP1156072).

\section{REFERENCES}

1. Grande I, Berk M, Birmaher B, Vieta E. Bipolar disorder. Lancet 2016;387:1561-72. https://doi.org/10.1016/S0140-6736(15)00241-X

2. Vieta E, Berk M, Schulze TG, Carvalho AF, Suppes T, Calabrese JR, et al. Bipolar disorders. Nat Rev Dis Primers 2018;4:18008. https://doi.org/10.1038/nrdp.2018.8

3. Ng F, Mammen OK, Wilting I, Sachs GS, Ferrier IN, Cassidy F, et al. The International Society for Bipolar Disorders (ISBD) consensus guidelines for the safety monitoring of bipolar disorder treatments. Bipolar Disord 2009;11:559-95. https://doi.org/10.1111/j.1399-5618.2009.00737.x

4. Malhi GS, Gershon S, Outhred T. Lithiumeter: Version 2.0. Bipolar Disord 2016;18:631-41. https://doi.org/10.1111/bdi.12455

5. Grof P, Duffy A, Cavazzoni P, Grof E, Garnham J, MacDougall M, et al. Is response to prophylactic lithium a familial trait? J Clin Psychiatry 2002;63:942-7. https://doi.org/10.4088/JCP.v63n1013
6. Malhi, GS, Bassett D, Boyce P, Bryant R, Fitzgerald PB, Fritz K, et al. Royal Australian and New Zealand College of Psychiatrists clinical practice guidelines for mood disorders. Aust N Z J Psychiatry 2015;49:1087-206. http://doi.org/10.1177/0004867415617657

7. Ott M, Stegmayr B, Salander Renberg E, Werneke U. Lithium intoxication: Incidence, clinical course and renal function - a population-based retrospective cohort study. J Psychopharmacol 2016;30:1008-19. https://doi.org/10.1177/0269881116652577

8. Azab AN, Shnaider A, Osher Y, Wang D, Bersudsky Y, Belmaker RH. Lithium nephrotoxicity. Int J Bipolar Disord 2015;3:13. https://doi.org/10.1186/ s40345-015-0028-y

9. Batlle DC, von Riotte AB, Gaviria M, Grupp M. Amelioration of polyuria by amiloride in patients receiving long-term lithium therapy. N Engl J Med 1985;312:408-14. https://doi.org/10.1056/NEJM198502143120705 